\title{
ANALYTIC CONTINUATION OF DIAGONALS AND HADAMARD COMPOSITIONS OF MULTIPLE POWER SERIES*
}

\author{
BY \\ R. H. CAMERON AND W. T. MARTIN
}

1. Introduction. A problem that has always interested mathematicians is the extension of an analytic function defined by a power series outside of its region of convergence. The classical Hadamard theorem $\dagger$ furnishes one method of doing this for functions of one variable. For this theorem gives us information about the analytic continuation of $A(z)=\sum_{0}^{\infty} a_{n} z^{n}$ provided we can find a way of factoring the coefficients $a_{n}$ into products $b_{n} c_{n}$ such that $B(z)=\sum b_{n} z^{n}$ and $C(z)=\sum c_{n} z^{n}$ are functions whose analytic continuations are known. Even if we are not able to factor the $a_{n}$ in such a way, we may be able to find a known function $F(x, y)=\sum f_{m n} x^{m} y^{n}$ whose coefficient matrix $\left\{f_{m n}\right\}$ has $\left\{a_{n}\right\}$ as its diagonal $\left(f_{n n}=a_{n}\right)$. One of the results of the present paper furnishes information concerning the region of analyticity of $A(z)$ in terms of the region of analyticity of $F(x, y)$. In case $F(x, y)=B(x) C(y)$ this method reduces to the preceding.

If we pass now to higher dimensions, our general point of view suggests a number of different possibilities. For example, suppose that we wish to investigate the analytic continuation of $A(x, y)=\sum a_{m n} x^{m} y^{n}$. If we can find a known function $F\left(x_{1}, x_{2}, y_{1}, y_{2}, y_{3}\right)=\sum f_{m_{1} m_{2} n_{1} n_{2} n_{3}} x_{1}{ }^{m_{1}} x_{2}{ }^{{ }_{2} y_{2}} y_{1}{ }^{{ }^{n}} y_{2}{ }_{2}{ }^{{ }_{2}} y_{3}{ }^{{ }^{3}}$ for which $a_{m n}=f_{m m n n n}$, then simply by iterating our primary theorem we can draw conclusions concerning the analytic extension of $A$ from our knowledge of the analytic extension of $F$. Similarly we can obtain information concerning $A(x, y, z)=\sum a_{m n p} x^{m} y^{n} z^{p}$ if we can find a known function $F\left(x_{1}, x_{2}, y_{1}, y_{2}, z_{1}, z_{2}\right)$

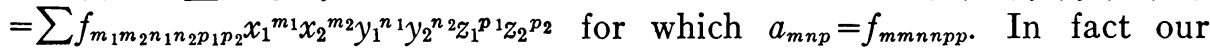
basic theorem can be iterated in such a way as to apply to any distribution of dimensions.

Just as our first result contains as a special case the classical Hadamard theorem, so these results contain an $n$-dimensional generalization of Hadamard's theorem. The regions we consider in the present paper are star-shaped and the nature of the singularities of the functions plays no role whatever.

* Presented to the Society, December 29, 1936; received by the editors May 26, 1937.

† J. Hadamard, Théorime sur les séries enticires, Acta Mathematica, vol. 22 (1898), pp. 55-64. 
Haslam-Jones* has obtained a two-dimensional analogue of the Hadamard theorem which furnishes information about a function $A(x, y)$ if there are two known functions $B(x, y), C(x, y)$ whose coefficients satisfy the relations $b_{m n} c_{m n}=a_{m n}$ and whose singularities satisfy certain conditions. If we compare his theorem with our two-dimensional theorem we see that neither includes the other. For our result does not always furnish as extensive an analytic continuation as that of Haslam-Jones, but it deals with a wider class of functions.

Grouping the terms into homogeneous polymonials, Bochner and Martin $\dagger$ have obtained an $n$-dimensional analogue of the Hadamard theorem. While (for $n>1$ ) their theorem does not deal directly with the individual coefficients, it does have the advantage of invariance under any affine transformation.

2. Stars. An important class of regions with which we shall be concerned in this paper is the class of star-shaped regions. $\ddagger$

Definition. A region $S$ in a space $R_{n}$ of $n$ complex variables $z_{1}, \cdots, z_{n}$ is called a $\left(p_{1}, \cdots, p_{n}\right)$-star if with the point $\left(z_{1}, \cdots, z_{n}\right)$ it contains also all the points $\left(\rho^{p_{1}} z_{1}, \cdots, \rho^{p_{n}} z_{n}\right)$, where $0 \leqq \rho \leqq 1$ and the $p_{i}$ are positive integers. In case $p_{1}=p_{2}=\cdots=p_{n}=p$, the $\left(p_{1}, \cdots, p_{n}\right)$-star is simply called a star.

It is clear that only the ratios of the p's are significant. It is also obvious that the union and intersection of two $\left(p_{1}, \cdots, p_{n}\right)$-stars are also $\left(p_{1}, \cdots, p_{n}\right)$ stars.

Notation. If $S$ is a $\left(p_{1}, \cdots, p_{n}\right)$-star in $R_{n}$, the symbol $S_{\left(z_{1} z_{2}\right)}$ will denote the point set consisting of all points $P\left(w, z_{3}, z_{4}, \cdots, z_{n}\right)$ in $R_{n-1}$ such that to $P$ there corresponds a continuous positive periodic function $r=r(\theta)$ of period $2 \pi$ for which each of the points $Q(\theta):\left(r e^{i \theta} w, r^{-1} e^{-i \theta}, z_{3}, \cdots, z_{n}\right)$ belongs to $S$, $(0 \leqq \theta \leqq 2 \pi)$.

We shall show that $S_{\left(z_{1} z_{2}\right)}$ is a $\left(p_{1}+p_{2}, p_{3}, \cdots, p_{n}\right)$-star. Let $P\left(w, z_{3}, \cdots, z_{n}\right)$ be a point of $S^{*} \equiv S_{\left(z_{1} z_{2}\right)}$ and let $r(\theta)$ be the associated function. Then since $S$ is a $\left(p_{1}, \cdots, p_{n}\right)$-star, each of the points

$$
\left(\rho^{p_{1}} e^{i \theta} w, \rho^{p_{2}} \gamma^{-1} e^{-i \theta}, \rho^{p_{3}} z_{3}, \cdots, \rho^{p_{n}} z_{n}\right), \quad(0 \leqq \rho \leqq 1,0 \leqq \theta \leqq 2 \pi),
$$

belongs to $S$. Thus if $R_{\rho}(\theta)=r(\theta) \rho^{-p_{2}}$, all of the points

$$
\left(\rho^{p_{1}+p_{2}} R_{\rho} e^{i \theta} w, R_{\rho}^{-1} e^{-i \theta}, \rho^{p_{3}} z_{3}, \cdots, \rho^{p_{n}} z_{n}\right), \quad(0<\rho \leqq 1,0 \leqq \theta \leqq 2 \pi),
$$

* U. S. Haslam-Jones, An extension of Hadamard's multiplication theorem, Proceedings of the London Mathematical Society, (2), vol. 27 (1928), pp. 223-232.

† S. Bochner and W. T. Martin, Singularities of composite functions in several variables, Annals of Mathematics, (2), vol. 38 (1937), pp. 293-302.

$\ddagger$ B. Almer, Sur quelques problèmes de la théorie des fonctions analytiques de deux variables complexes, Arkiv för Matematik, Astronomi och Fysik, vol. 17 (1922), pp. 1-70; W. T. Martin, Special regions of regularity of functions of several complex variables, Annals of Mathematics, (2), vol. 38 (1937), pp. 602-625. 
are in $S$, and hence all of the points

are in $S^{*}$.

$$
\left(\rho^{p_{1}+p_{2}} w, \rho^{p_{3}} z_{3}, \cdots, \rho^{p_{n} z^{n}}\right), \quad(0<\rho \leqq 1),
$$

It only remains to show that $S^{*}$ is an open set, as connectedness obviously follows from the last statement. (It is trivial to show that $\left(w=0, z_{3}=0, \cdots\right.$, $z_{n}=0$ ) belongs to $S^{*}$ since $S$ contains the origin.) To show that $S^{*}$ is open, consider a point $P_{0}\left(w^{(0)}, z_{3}{ }^{(0)}, \cdots, z_{n}{ }^{(0)}\right)$ of it. Since $S$ is open, with $\left(r e^{i \theta} w^{(0)}\right.$, $\left.r^{-1} e^{-i \theta}, z_{3}{ }^{(0)}, \cdots, z_{n}{ }^{(0)}\right)$ there is contained an $n$-dimensional neighborhood $N(\theta)$ of $Q_{0}(\theta)$ in $S$,

$$
\begin{aligned}
& N(\theta): \quad\left|z_{1}-r e^{i \theta} w^{(0)}\right|<\epsilon(\theta), \quad\left|z_{2}-r^{-1} e^{-i \theta}\right|<\epsilon(\theta), \quad\left|z_{j}-z_{j}^{(0)}\right|<\epsilon(\theta), \\
& (j=3, \cdots, n) \text {. }
\end{aligned}
$$

Because of the continuity of $r(\theta)$ it is clear that there is a positive $\epsilon$ independent of $\theta$ such that for all $\theta$ in $0 \leqq \theta \leqq 2 \pi$ all of the points for which $\left|z_{1}-r e^{i \theta} w^{(0)}\right|<\epsilon$, $\left|z_{2}-r^{-1} e^{i \theta}\right|,\left|z_{j}-z_{j}{ }^{(0)}\right|<\epsilon,(j=3, \cdots, n)$, belong to $S$. Let

Thus when

$$
\delta=\min \left\{\frac{\epsilon}{\max r(\theta)}, \epsilon\right\} \text {. }
$$

$$
\left|w-w^{(0)}\right|<\delta,\left|z_{3}-z_{3}{ }^{(0)}\right|<\delta, \cdots,\left|z_{n}-z_{n}^{(0)}\right|<\delta,
$$

the point $\left(r e^{i \theta} w, r^{-1} e^{-i \theta}, z_{3}, \cdots, z_{n}\right)$ is in the $\epsilon$-neighborhood of $Q_{0}(\theta)$ and hence in $S$. Thus $S^{*}$ is a $\left(p_{1}+p_{2}, p_{3}, \cdots, p_{n}\right)$-star. We shall call $S^{*} \equiv S_{\left(z_{1} z_{2}\right)}$ a contracted star of $S$. More general contractions will later be given.

Definition. Let $f\left(z_{1}, \cdots, z_{n}\right)$ be any function analytic in a neighborhood of the origin. Let us define a point set $S \equiv S\left(f ; p_{1}, \cdots, p_{n}\right)$ as follows: A point $\left(z_{1}, \cdots, z_{n}\right)$ belongs to $S$ if $f$ is regular at all of the points $\left(\rho^{p_{1}} z_{1}, \cdots, \rho^{p_{n}} z_{n}\right)$, $0 \leqq \rho \leqq 1$. It is clear that $S$ is a $\left(p_{1}, \cdots, p_{n}\right)$-star; we shall call $S$ the $\left(p_{1}, \cdots, p_{n}\right)$-star of $f$. We shall denote by $S(f)$ the $(1, \cdots, 1)$-star of $f$.

3. Proof of the central theorem. We shall now prove the following extension of the Hadamard theorem on which our remaining results will be based:

THEOREM 1. Let

$$
A\left(z_{1}, \cdots, z_{n}\right)=\sum a_{\mu_{1} \cdots \mu_{n}} z_{1}^{\mu_{1}} \cdots z_{n}^{\mu_{n}}
$$

be analytic in a neighborhood of the origin, and let $S$ be its $\left(p_{1}, \cdots, p_{n}\right)$-star. Then the diagonal function

$$
A_{\left(z_{1} z_{2}\right)}\left(w, z_{3}, \cdots, z_{n}\right)=\sum a_{m m \mu_{3}} \cdots \mu_{n} w^{m} z_{3}^{\mu_{3}} \cdots z_{n}^{\mu_{n}}
$$

is analytic in the contracted star $S^{*} \equiv S_{\left(z_{1} z_{2}\right)}$. 
For the proof let $P_{0}\left(w^{(0)}, z_{3}{ }^{(0)}, \cdots, z_{n}{ }^{(0)}\right)$ be any point in $S^{*}$. Then by definition of $S^{*}$ there exists a continuous positive periodic function $r(\theta)$ $=r\left(\theta ; P_{0}\right)$ such that all of the points

$$
\left(r e^{i \theta} w^{(0)}, r^{-1} e^{-i \theta}, z_{3}^{(0)}, \cdots, z_{n}^{(0)}\right), \quad 0 \leqq \theta \leqq 2 \pi,
$$

are in $S$. Since $S$ is open it is clear that for sufficiently small positive $\delta$ all the points $\left(r^{*} e^{i \theta} w^{(0)}, r^{*-1} e^{-i \theta}, z_{3}{ }^{(0)}, \cdots, z_{n}{ }^{(0)}\right)$ are in $S$, where

$$
r^{*}=r^{*}\left(\theta ; P_{0}\right)=\frac{1}{\delta} \int_{\theta}^{\theta+\delta} r(\phi) d \phi .
$$

Then the set of points $t=r^{*}(\theta) e^{i \theta}$ forms a rectifiable closed curve $C^{*}=C^{*}\left(P_{0}\right)$ around the origin. For $t$ on $C^{*}$ and $\left(w, z_{3}, \cdots, z_{n}\right)$ in a sufficiently small neighborhood $N=N_{\epsilon}\left(P_{0}\right)$ of $P_{0}$,

$N_{\epsilon}\left(P_{0}\right): \quad\left|w-w^{(0)}\right|<\epsilon,\left|z_{3}-z_{3}{ }^{(0)}\right|<\epsilon, \cdots,\left|z_{n}-z_{n}{ }^{(0)}\right|<\epsilon$,

the function $A\left(w t, t^{-1}, z_{3}, \cdots, z_{n}\right)$ is analytic in all its variables. Then since $S$ is a $\left(p_{1}, \cdots, p_{n}\right)$-star, it follows that $A\left(u^{p_{1} w t}, u^{p_{2}} t^{-1}, u^{p_{3} z_{3}}, \cdots, u^{p_{n}} z_{n}\right)$ is analytic in $w, z_{3}, \cdots, z_{n}, t, u$ for $P\left(w, z_{3}, \cdots, z_{n}\right) \varepsilon N\left(P_{0}\right), t_{\varepsilon} C^{*}, 0 \leqq u \leqq 1$. Thus

(3) $\quad g\left(w, z_{3}, \cdots, z_{n}, u ; P_{0}\right)=\frac{1}{2 \pi i} \int_{C^{*}\left(P_{0}\right)} A\left(u^{\left.p_{1} w t, u^{p_{2}} t^{-1}, u^{p_{3} z_{3}}, \cdots, u^{p_{n}} z_{n}\right)} \frac{d t}{t}\right.$

is analytic in $w, z_{3}, \cdots, z_{n}, u$ when $P \varepsilon N$ and $0 \leqq u \leqq 1$. Now let $R$ be positive and such that the series (1) converges whenever $\left|z_{j}\right|<R,(j=1, \cdots, n)$, and let $\gamma$ and $\Gamma$ be the minimum and maximum values of $|t|$ for $t$ on $C^{*}$. Let

$$
\delta_{1}=\min \left\{\left[\frac{R}{\left(\left|w_{1}{ }^{(0)}\right|+\epsilon\right) \Gamma}\right]^{1 / p_{1}}, \quad(R \gamma)^{1 / p_{2}},\left[\frac{R}{\left|z_{j}^{(0)}\right|+\epsilon}\right]^{1 / p_{j}}\right\},
$$

for $j=3, \cdots, n$. Then if $0 \leqq u<\delta_{1}$, and $F_{\varepsilon} N$,

$$
\begin{aligned}
& g\left(w, z_{3}, \cdots, z_{n}, u ; P_{0}\right)
\end{aligned}
$$

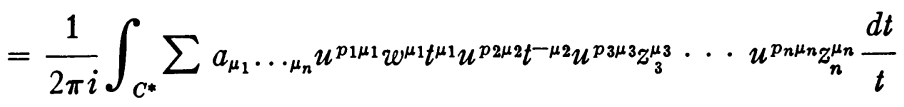

$$
\begin{aligned}
& =\sum_{m, \mu_{3}, \cdots, \mu_{n}=0}^{\infty} a_{m m \mu_{3} \cdots \mu_{n}} u^{\left(p_{1}+p_{2}\right) m+p_{3} \mu_{3}+\cdots+p_{n} \mu_{n}} w^{m} z_{3}^{\mu_{3}} \cdots z_{n}^{\mu_{n}} \\
& =A_{\left(z_{1} z_{2}\right)}\left(u^{p_{1}+p_{2}} w, u^{p_{3}} z_{3}, \cdots, u^{p_{n}} z_{n}\right) \\
& =\sum_{k=0}^{\infty} u^{k}\left\{\sum_{\left(p_{1}+p_{2}\right) m+p_{3} \mu_{3}+\cdots+p_{n} \mu_{n}=k} a_{m m \mu_{3} \cdots \mu_{n}} w^{m} z_{3}^{\mu_{3}} \cdots z_{n}^{\mu_{n}}\right\} \\
& =\sum_{k=0}^{\infty} B_{k}(P) u^{k} \text {. }
\end{aligned}
$$


Since for a fixed $P_{\varepsilon} N$ this is an analytic function of $u$ for $0 \leqq u \leqq 1$, its value at $u=1$ is given by the Le Roy $\dagger$ sum of the last series. Thus

$$
g\left(P, 1 ; P_{0}\right)=g\left(w, z_{3}, \cdots, z_{n}, 1 ; P_{0}\right)=\lim _{s \rightarrow 1^{-}} \sum_{k=0}^{\infty} \frac{\Gamma(k s+1)}{\Gamma(k+1)} B_{k}(P) .
$$

Since $g\left(P, u ; P_{0}\right)$ is analytic for $P_{\varepsilon} N, 0 \leqq u \leqq 1, g\left(P, 1 ; P_{0}\right)$ is certainly analytic for $P_{\varepsilon} N$. But the summation in (5) is independent of $P_{0}$, and in particular it is defined when $P=P_{0}$, while $P_{0}$ may be any point in $S^{*}$. Thus

$$
\tilde{A}(P)=\lim _{s \rightarrow 1^{-}} \sum_{k=0}^{\infty} \frac{\Gamma(k s+1)}{\Gamma(k+1)} B_{k}(P)
$$

is defined throughout $S^{*}$; and since $g\left(P, 1, P_{0}\right)=\tilde{A}(P)$ throughout some $N_{0}\left(P_{0}\right)$, it follows that $\tilde{A}(P)$ is regular at all points of $S^{*}$. Since the Le Roy method of summation is regular, it follows that

$$
\tilde{A}(P)=\sum_{k=0}^{\infty} B_{k}(P)=A_{\left(z_{1} z_{2}\right)}(P)
$$

for $P$ sufficiently close to the origin. Thus our theorem is proved.

4. Scope of Theorem 1. As an indication of the scope of Theorem 1, we shall show that it contains the classical Hadamard theorem as a special case. Indeed, if $A\left(z_{1}, z_{2}\right)=B\left(z_{1}\right) C\left(z_{2}\right)$, where $B$ and $C$ are any two functions analytic in the neighborhood of this origin, then the diagonal function $A_{\left(z_{1} z_{2}\right)}$ is the Hadamard composite of $B$ and $C$. Thus if $\xi_{\theta} e^{i \theta}$ and $\eta_{\theta} e^{i \theta}$ are the star vertices of $B$ and $C$, it is only necessary to show that whenever

$$
0 \leqq c<\underset{0 \leqq \phi \leqq 2 \pi}{\text { g. } 1 . \mathrm{b} .} \xi_{\gamma+\phi} \eta_{-\phi}
$$

then the point $c e^{i \gamma}$ belongs to $S_{\left(z_{1} z_{2}\right)}$, and hence by Theorem 1 is a regular point of $A_{\left(z_{1} z_{2}\right)}$. We shall show this by proving the existence of a continuous positive periodic function $r(\theta)$ such that for all $\theta$

$$
\operatorname{cr}(\theta)<\xi_{\gamma+\theta} ; \quad \frac{1}{r(\theta)}<\eta_{-\theta} .
$$

In the first place it follows from (6) that the set $\mathscr{B}$ of points $w=t e^{i \theta}$ defined by the inequalities

$$
\frac{1}{\eta_{-\theta}}<t<\frac{\xi_{\theta+\gamma}}{c}
$$

† See for example, L. L. Smail, Theory of Summable Infinite Processes, Oregon, 1925, pp. 13, 14. 
contains points on each ray arg $w=\theta$. Also due to the continuity of the functions $z^{-1}$ and $c^{-1} z$ and the fact that $S(B)$ and $S(C)$ are open regions, the set $\mathscr{B}$ is an open region. It is obvious that we can find a $\theta$-neighborhood of any $\theta$ throughout which there exists a positive continuous function $r_{1}(\theta)$ which satisfies (7). Thus by the Heine-Borel theorem there is a positive periodic function $r_{2}(\theta)$ which has at most a finite number of finite jumps in each period interval and which always satisfies (7). Due to (8) we can join the ends of the jumps and obtain a multiple-valued function satisfying (7); and since $\mathfrak{B}$ is open we can modify this function so as to make it single-valued. Thus $r(\theta)$ exists and $c e^{i \gamma}$ belongs to $S_{\left(z_{1} z_{2}\right)}$.

5. Generalizations. Since the contraction of a star is a star, the process of contraction may be iterated. In order to have the resulting stars as extensive as possible, and independent of the order, we make the following definition:

Definition. If $S$ is a $\left(p_{1}, \cdots, p_{n}\right)$-star, and if

$$
T=S_{\left(z_{1} z_{2}\right)}, \quad U=S_{\left(z_{3} z_{4}\right)},
$$

then we define

$$
S_{\left(z_{1} z_{2}\right)\left(z_{3} z_{4}\right)}=T_{\left(z_{z_{4}}\right)}+U_{\left(z_{1} z_{2}\right)}
$$

where the sum is a union in the sense of point sets. It is clear that it is a $\left(p_{1}+p_{2}, p_{3}+p_{4}, p_{5}, \cdots, p_{n}\right)$-star. Similarly we define

$$
S_{\left(z_{1} z_{2}\right)\left(z_{z} z_{4}\right)\left(z_{6} z_{6}\right)}=\left[S_{\left(z_{1} z_{2}\right)\left(z_{8} z_{4}\right)}\right]_{\left(z_{6} z_{6}\right)}+\left[S_{\left(z_{1} z_{2}\right)\left(z_{6} z_{6}\right)}\right]_{\left(z_{8} z_{4}\right)}+\left[S_{\left(z_{z} z_{4}\right)\left(z_{6} z_{6}\right)}\right]_{\left(z_{1} z_{2}\right)}
$$

and

$$
S_{\left(z_{12} z_{z}\right)}=T_{\left(z_{12} z_{3}\right)}+U_{\left(z_{18} z_{2}\right)}+V_{\left(z_{28} z_{1}\right)}
$$

where

$$
T=S_{\left(z_{1} z_{2}\right)}, \quad U=S_{\left(z_{1} z_{3}\right)}, \quad V=S_{\left(z_{2} z_{z}\right)} .
$$

Contractions of higher orders are defined by the obvious extension of these definitions.

THEOREM 2. Let (1) be analytic in a neighborhood of the origin and let $S=S\left(f ; p_{1}, \cdots, p_{n}\right)$ be its $\left(p_{1}, \cdots, p_{n}\right)$-star. Then the diagonal functions

$$
A_{\left(z_{1} z_{2}\right)\left(z_{3} z_{4}\right)}\left(z_{12}, z_{34}, z_{5}, \cdots, z_{n}\right)=\sum a_{m m q \mu_{5}} \cdots \mu_{n} z_{12}^{m} z_{32}^{q} z_{5}^{\mu_{5}} \cdots z_{n}^{\mu_{n}}
$$

and

$$
A_{\left(z_{1} z_{2} z_{8}\right)}\left(z_{123}, z_{4}, \cdots, z_{n}\right)=\sum a_{m m m \mu_{4}} \cdots \mu_{n} z_{123}^{m} z_{4}^{\mu_{4}} \cdots z_{n}^{\mu_{n}}
$$

are analytic in the stars $S_{\left(z_{1} z_{2}\right)\left(z_{3} z_{4}\right)}$ and $S_{\left(z_{1} z_{2} z_{3}\right)}$ respectively. 
For the proof we note that

and

$$
A_{\left(z_{1} z_{2}\right)\left(z_{3} z_{4}\right)}=\left[A_{\left(z_{1} z_{2}\right)}\right]_{\left(z_{3} z_{4}\right)}=\left[A_{\left(z_{3} z_{4}\right)}\right]_{\left(z_{1} z_{2}\right)},
$$

$$
A_{\left(z_{1} z_{2} z_{3}\right)}=\left[A_{\left(z_{1} z_{2}\right)}\right]_{\left(z_{12} z_{3}\right)}=\left[A_{\left(z_{1} z_{3}\right)}\right]_{\left(z_{13} z_{2}\right)}=\left[A_{\left(z_{2} z_{3}\right)}\right]_{\left(z_{23} z_{1}\right)} .
$$

The theorem follows by iteration of Theorem 1 .

6. Two-dimensional Hadamard theorem. We can now obtain as a special case of Theorem 2 a two-variable extension of the Hadamard theorem. Let us take a specialized function of four variables; namely, a product of two functions each of two variables,

$$
\begin{aligned}
A(s, u, t, v) & =B(s, t) C(u, v), \\
B(x, y) & =\sum b_{m n} x^{m} y^{n}, \\
C(x, y) & =\sum c_{m n} x^{m} y^{n} .
\end{aligned}
$$

Then our previously defined diagonal function (see (9)) becomes

$$
A_{(s u)(t v)}(x, y)=\sum b_{m n} c_{m n} x^{m} y^{n}
$$

and our special theorem yields the following theorem:

THEOREM 3. Let (12) and (13) be any two functions analytic in a neighborhood of the origin, and let $S(B)$ and $S(C)$ be their stars. Then the Hadamard composition

$$
H(x, y)=\sum b_{m n} c_{m n} x^{m} y^{n}
$$

is analytic in the star $S^{*}=S_{1}+S_{2}$, where $S_{1}$ and $S_{2}$ are defined in terms of $S(B)$ and $S(C)$ as follows. $A$ point $(x, y)$ is in the star $S_{1}$ if for all $\phi, \theta$ the points

are in $S(B)$ and the points

$$
\left(x r_{\phi}(\theta) e^{i \theta}, y r^{\prime}(\phi) e^{i \theta}\right)
$$

$$
\left(\frac{1}{r_{\phi}(\theta) e^{i \theta}}, \frac{1}{r^{\prime}(\phi) e^{i \theta}}\right)
$$

are in $S(C)$, where $r^{\prime}(\phi)$ is a positive continuous periodic function of $\phi$, and $r_{\phi}(\theta)$ is a positive periodic continuous function of $\theta$ and depends also on the parameter $\phi$. The star $S_{2}$ is similarly defined, with the roles of $r$ and $r^{\prime}$ interchanged.

From the statement of Theorem 3 and the manner in which it follows from our general theorem, one could easily obtain an $n$-variable extension of the Hadamard theorem.

Massachusetts Institute of Technology,

Cambridge, Mass. 